\title{
Understanding qualitative drivers in distance collaboration for architectural services
}

\author{
B. Gardiner ${ }^{1}$, P. Tombesi ${ }^{1}$, B. Dave ${ }^{1}$ \& P. Scriver ${ }^{2}$ \\ ${ }^{1}$ University of Melbourne, Australia \\ ${ }^{2}$ University of Adelaide, Australia
}

\begin{abstract}
Enhanced global connectivity, networks' capacity to carry data, and increases in transmission speed already affect the way architectural practices work. With computer-assisted drafting (CAD) equipment now used by the overwhelming majority of architectural offices globally, electronic transfer of drawings is on the rise, followed by opportunities to benefit from the economic advantage of digital technologies by setting up remote links. Researchers in areas such as the media, software engineering, accounting, and light manufacturing have examined the industrial, cultural and regional development underpinnings of such phenomena. By contrast, very few analyses exist in architecture that link technological opportunities to social transformations, and technical skills to market development. Our research responds to this challenge by investigating qualitative differences in the performance of distant actors, and aims to determine whether these differences can be related to environmental characteristics.
\end{abstract}

Keywords: outsourcing, architectural firms, drafting services.

\section{Introduction}

Enhanced global connectivity, networks' capacity to carry data, and increases in transmission speed already affect the way architectural practices work. With computer-assisted drafting (CAD) equipment now used by the overwhelming majority of architectural offices globally, electronic transfer of drawings is on the rise, followed by opportunities to benefit from the economic advantage of digital technologies by setting up remote links [1,2]. Researchers in areas such as the media, software engineering, accounting, and light manufacturing have 
examined the industrial, cultural and regional development underpinnings of such phenomena $[3,4]$. By contrast, very few analyses exist in architecture that link technological opportunities to social transformations, and technical skills to market development. Our research responds to this challenge by investigating qualitative differences in the performance of distant actors, and aims to determine whether these differences can be related to environmental characteristics.

The division of labour between design and technical production has been a perennial aspect of architectural project organization. The acceptance of computer-assisted drafting equipment and the sophistication of electronic communication has facilitated this division of production globally. However, little information beyond the anecdotal is available about the qualitative performances of offshore architectural production. Unless one has participated in such ventures, it is difficult to assess the potential and viability of geographically distant outsourcing.

Supported by an Australian Research Council grant in 2005, a research program was established, which proposed a series of controlled documentation collaborations between seven Melbourne-based architectural offices and four types of service documentation sub-contractors. The project seeks to: 1) identify potential difficulties faced in establishing collaborative linkages; 2) identify factors that impact on collaboration viability; 3) identify indicators for the demonstration of skills within three tacit practice arenas. These arenas being: vocational (ability to simply act as a drafting agent, by translating information into construction drawing formats), professional (understanding and supporting the design intent and implied design language, tracking down possible inconsistencies; and adding or correcting incomplete information) and sociocultural (regulatory requirements, codes of practice, technical traditions that influence the selection of details; and project procurement practices and the structure of documents).

Through this process, we hope to develop an inclusive conceptual scaffold that allows the successful parameters of digitally supported distant collaborations to be characterized, and their likely impact on forms of architectural practice to be weighed up [5]. The aim is to articulate the conditions that should be satisfied for Australian architectural establishments to outsource their work, and to determine whether or not the different types of firms involved in the market of digital collaborations at the moment have the technical capacity and operational synergies for effective alliances. This will lead to an evaluation of the industrial potential of distant collaboration between architectural providers, and the assessment of the likelihood that such practice will develop into a fully-fledged mode of service delivery [6].

This paper reports the results from the initial stages of our research experiments, data collection and preliminary findings arising from a pilot project. 


\section{Project structure}

Seven Melbourne-based firms were chosen to reflect the profile of the local profession in terms of professional markets, practice size and work focus. In consultation with each practice a project was chosen that typified the practice's working platforms. These projects form the basis of a series of investigations that will compare the performance of four types of documentation service providers:

- Australian firms specializing in contract documentation, thereby reflecting local market labour division;

- Web-based firms; set-up to work remotely and take advantage of technological opportunities;

- Indian based professional firms; with similar historical roots but different socio-economic and environmental conditions;

- South-East Asian firms, with a staff profile in Australian education and professional practice.

The proposed brief is for the service providers to produce a sample set of seven different construction drawings derived from the schematic and design development drawings of a completed project of the Melbourne-based practice. The task is to interpret and convert schematic drawings and preliminary information into a potential construction drawing set. The sample series of documentation is representative of location, assembly and component drawing arrays that engage with the project at different representative scales.

The first stage of the research program has been to establish a pilot project to understand and test the operative conditions that would need to be confronted and resolved prior to the full project study. For the pilot it was decided to use one of the most common project types of Australian architectural practice - the single family house. The project embedded a series of technical tests and design resolution tasks as the building is a modernist design unit, placed at the high-end of the domestic market incorporating quasi-industrial materials and construction systems (e.g. concrete and commercial aluminum window sections).

The Australian architectural practice had produced a higher than average amount of highly resolved construction documents, many of which were relational in nature to fully explain the integration of services, finishes and joinery units. Location drawings were set up as a series of layers effectively trade sequencing structure, services and finishes in order to minimize the potential for positional errors due to the use of concrete as the structural and aesthetic generator of the architecture. In addition, resolution of structural engineering, regulatory controls, services incorporation and building performance issues necessitated careful detailing in order to ensure the fine tolerances of finish demanded by a building design of deceptively simple but precise lines. In fact, it was during the design documentation that the architectural practice invested most of its skilled-labour.

The interest therefore lay in testing the ability of a collaborative documentation partner to coalesce with this working method. For this reason, the proposed documentation drawing test included four plan drawings, three of 
which represented the building at different levels; one additional plan which indicated the layering methodology dividing structure and finish; one critical representational elevation and section drawing; one drawing of vertical construction details linked back to the section and one drawing of vertical and horizontal window details which were crucial to achieve the design aesthetic proposed. The selection of these documents was deliberate, to expose:

- The understanding of the design intent and its consistency of interpretation through various modes of representation;

- The resolution of technical issues and the consideration of these issues through the different levels of the building;

- The familiarity with and understanding of Australian building regulatory regimes;

- The ability to deal with local codes of practice by including a specific request for the proposal and technical resolution of a drainage layout in compliance with an Australian Standard industry code. The layout required consideration for its integration with the building aesthetic and the implied condition for the concealment of all services and forethought of potential construction conflicts with the design intent;

- The ability to understand, interpret and communicate proprietary systems, and resolve these within the design schema.

The challenge, here, was to understand and represent subtle variations in the configuration of each system vis-à-vis its position in space and within the building whilst supporting both semantic and performance value of the solution designed by the architectural practice. It also afforded the ability to gauge the response to the firm's specific language decisions (i.e. prescriptive technology), interpretation of regulatory requirements (i.e. normative technology) and understanding of local traditions (i.e. conventional technology).

To facilitate the production of these drawings a project information package was compiled that consisted of:

- a series of preliminary generating design sketches for the project;

- a series of images that were used as reference sources for the design intent;

- a cad file site survey drawing, defining property boundaries and site levels;

- a pdf set of regulatory approved town planning drawings;

- a complete set of 'Autocad' design development drawings; and which would form the cad drawing basis for the proposed documentation set;

- a series of construction detail sketches to communicate the key assembly components;

- a description of the principal construction elements and a list of proprietary systems with limited accompanying trade literature;

- a cad file set-up of the architectural practice's documentation protocols (layer and line types, title sheet set-up); 
- a pdf copy of a complete set of construction documentation drawings from another project, which would be used to define the architectural practice's documentation communication strategies;

- $\quad$ a set of instructions on the seven required sample drawing set to be produced with a specific requirement for a proposal for a roof drainage design in accordance with a particular Australian code of practice, fig 2;

- a list of the proposed total construction drawing set for the project numbering seventy-one drawings.
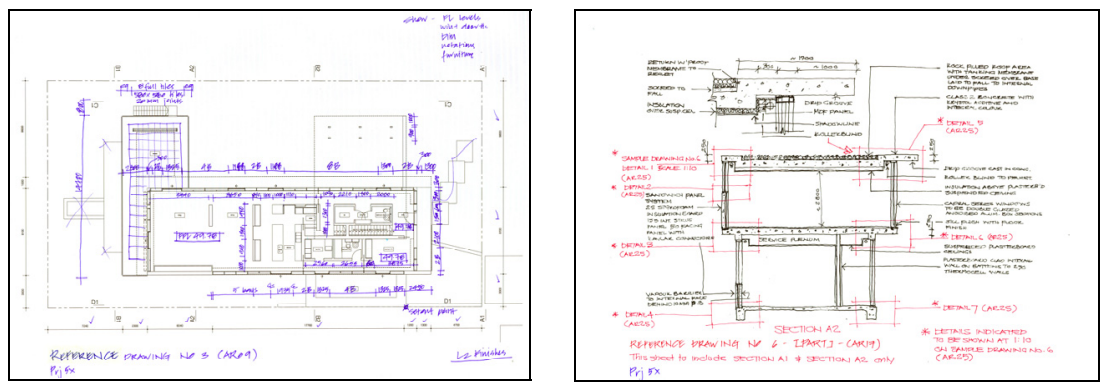

Figure 1: Sample of sketch and design development drawings provided. (Source: Authors, based on the Australian practice's drawings.)

It was decided to direct the pilot project to a limited number web-based firms first, in order to determine the potential take-up rate, anticipated cost, market condition and operational procedures. In effect, what would happen if one blindly went out to generically test the waters of web-based documentation service providers?

\section{Project process}

Web-based firms were sourced from their exposure in print media, through internet based search engines and by referral. The final selection of firms was based on the web-site descriptions of their service capabilities, practice capacity, experience with off-shore alliancing and English language capabilities. Following this process, a list of eighteen firms was collated. Of these firms: ten identified their geographic base as being in India, three in the Philippines, two in the United States, with one each from Mexico, Botswana and the United Kingdom.

An off-shore electronic website was established and through this facility each firm was forwarded an electronic mail invitation to participate in a pilot test, to investigate the suitability of remote documentation services to a selection of Australian architectural practices.

The firms were invited to register their interest in providing such services, with a request to forward information on firm profile, contact details, references, payment conditions, quality assurance procedures and confidentiality 
arrangements. The invitation outlined that through a secure server the project information package was available with a request to translate the information into construction documents in Autocad Dwg format and to price, on the basis of the scope of work identified, the production of the limited set of drawings as well as the entire documentation package and to indicate the turnover time anticipated for both the test and the complete documentation package.

Of the eighteen firms five responded immediately and downloaded the information package, three were based in India, one from Botswana and one from the Philippines. One of the Indian firms proposed working on the project on the basis of hourly rates that were approximately $26 \%$ of the Melbourne practice; however, they did not proceed beyond this communication. Another Indian based firm (Firm 1) and the Philippines firm (Firm 2) proceeded further by providing the requested service provider information and followed with engagement terms.

Firm 1 identified that their client base was primarily in the USA, with some clients from the UK and Germany. They had not worked with an Australian client and were not familiar with Australian regulatory frameworks. Their core services were in the transference of conceptual sketches into base CAD drawings and the production of CD sets from DD drawings for a range of project types including residential, corporate, education and retail work. They presented ISO 9001-2000 certification as evidence of their quality assurance system. No information on staff numbers or capacity were provided, except a brief profile of six unnamed personnel, five of whom held architectural degrees from Indian institutions with one identified as a consulting architect with US practice experience.

Firm 2 identified that their client group was primarily Philippine based, although they had current projects in California and had completed projects in Shanghai. They lacked experience with Australian regulatory frameworks, with core services being in full scope construction documentation and presentation rendering. No information was provided on their quality assurance procedures. They were a small office, identifying by name the two architect principals and seven staff members, one of whom is an architect.

Fee proposals were such that the quote from Firm 2 was $47 \%$ of Firm 1 . Both requested a down-payment, with Firm 1 requiring an advance of $50 \%$ of the fee up-front prior to proceeding. Both firms were engaged to participate in the pilot exercise.

Both firms responded with the complete set of drawings within twelve days. During this period Firm 1 issued an intermediary set for review with minimal queries and Firm 2 issued two intermediary sets with a number of queries, although not extensive quite specific in their content. Firm 1 requested payment to cover the costs of purchasing the Australian code that the project instructions required.

The drawing sets provided indicated that neither firm had difficulty in setting up the required cad standards and that layering protocols and documentation transfer were not a point of issue. Neither firm established clear and well defined project management protocols. The process of managing project 
communication and requests for information were loose and necessitated the implementation of a system to control these aspects of project delivery.

Neither firm proposed the development of the documentation beyond the minimal requirements of the tasks set. Detailed resolution of important design and construction aspects were cursory and unresolved. Significantly, there were important construction details dictated by the design that were not picked up, these included issues dealing with waterproofing, provision for movement and the treatment of finishes.

Two examples are presented as indicators of the vocational, professional and socio-cultural evaluative categories to the pilot responses. The first is a selected overview of the vertical sectional assembly detail drawing, fig. 2; the second being the response to a proposal for a roof drainage layout.

Vocational evaluation centred on the ability of the firm to transfer information into construction drawing notational formats. Firm 1 had a greater number of inaccuracies such as missing lines, lack of drawing legibility with consideration to drawing formatting, poor cross-referencing, as well as notational and dimensional inaccuracies.

Professional evaluation centred on understanding and supporting the design intent and implied design language. This is best exemplified in both firms response to the request to propose a roof drainage layout in compliance with an Australian standard code of practice. Neither firm outlined how they interpreted the stipulated code requirements for roof drainage, with Firm 2 initially proposing a drainage system that did not account for the construction system or important design imperatives and issued drawings indicating exposed downpipes along a visually prominent fully glazed façade. They, however, responded to a request for the concealment of these drainage outlets by a well resolved solution and provided additional explanatory relational drawings to indicate how the system was placed vertically through the building. Firm 1 proposed a system that potentially altered one of the important design intents of minimising the concrete roof edge profile. The proposal did not account for structure, embedding vertical drainage within precast concrete wall panels and failed to facilitate the required ceiling levels demanded by the design aesthetic.

Socio-cultural evaluations embraced technical traditions, regulatory requirements and the structuring of the documents. Both firms did not display high levels of engagement with these issues. Detail drawing sheet layouts were confusing and counter to the implied approach suggested through sketch information. Firm 1 proposed glazing profiles that were counter to specifications, with Firm 2 relying on information requests rather than sourcing available technical material. Firm 1 displayed poor understanding of proprietary systems and documented information that generated internal conflicts within their own drawings.

Both firms displayed attributes in drawing conversion capacity but lacked proficiency at tasks that required high order capacity in vocational, professional and socio-cultural considerations. The process also suggested that the design development drawings were in fact already highly resolved and therefore major errors and discrepancies were not overt. 
162 Digital Architecture and Construction

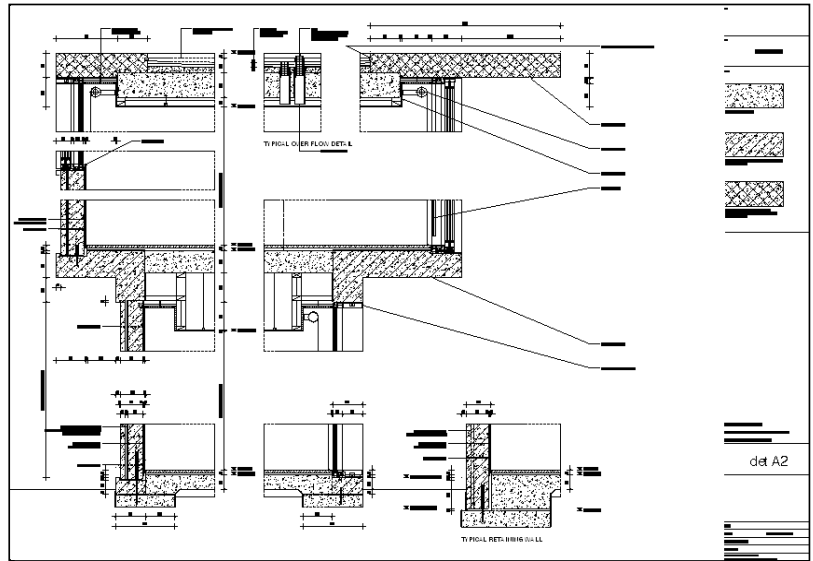

(a)

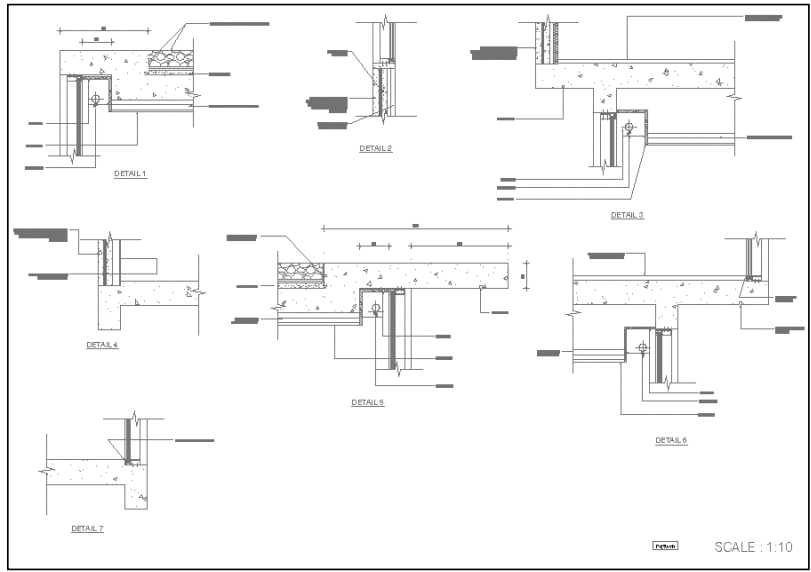

(b)

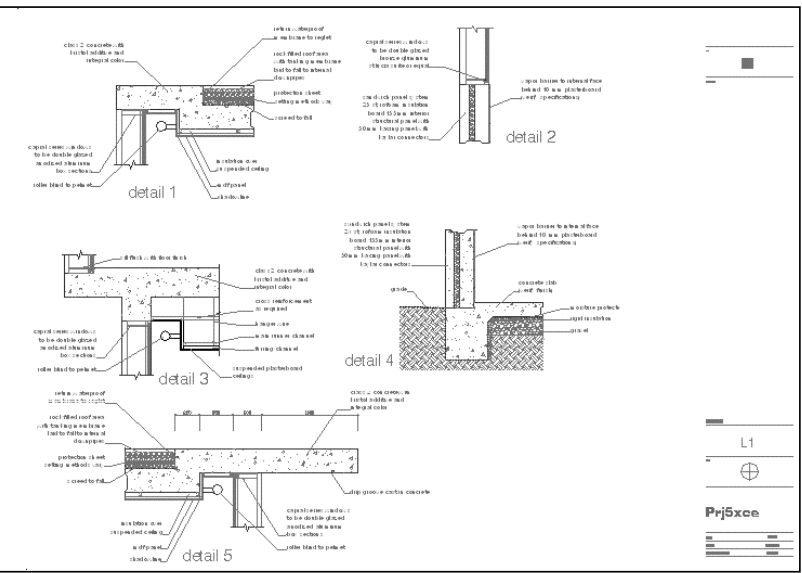

(c)

Figure 2: $\quad$ Detail drawing sheet. (a) Actual project (Source: Australian based practice); (b) Firm 1 (Source: Commissioned by authors); (c) Firm 2 (Source: Commissioned by authors). 


\section{Discussion}

Although the pilot study proved to be a limited sample it still provided the opportunity for a series of speculations to be proposed and exposed a number of issues that will inform the next phase of the investigation.

\subsection{Low response rate}

One may speculate as to why the take-up rate was so low with only $28 \%$ of invitees responding and only $11 \%$ continuing to proceed to participate in the test project. It may well be that the firms were not yet committed or geared to offshore transactions. The cost of establishing a web-based profile is relatively low, with no risk attached in creating a web profile and using it to gauge market interest. There was also no ability to gauge whether the firms were already operating or still operating in the form presented, nor could it be determined how long they had been operating in the way they had presented themselves on the web.

Was there was a mismatch between the advertised capabilities and the proposed task? Were the firms geared towards conventional drafting or presentation packaging as against design and technical resolution? Did the task require a greater level of interpretive expertise or a higher level of technical sophistication beyond the in-house capacity of the firm and therefore the project was not of commercial interest? Did the test in requiring information on experience with Australian regulatory frameworks preclude those firms who lacked such experience and the entrepreneurial will to pursue the opportunity presented? Was it simply as one respondent advised that they were just "too busy"?

The form of approach may also have led to a low acceptance rate, as the offer was presented in terms of a brokerage arrangement. The positioning of an intermediary removed the opportunity to enter into a direct commercial relationship with the potential actual client and may have lessened interest in proceeding to engage with the pilot test as proposed. The firms may already have established markets and are less interested or have no need to go through intermediate steps.

Such speculations and questions demonstrate the need for research into outsourcing to include the operative conditions that prevail from the service provider perspective. It highlights the difficulty in identifying appropriate service partners. In order to cultivate particular relationships there is a risk in adopting a generic approach and much greater specificity is required. Further stages of the research will include investigating the performance of web-based service providers where face-to-face contact and preliminary capacity reviews have been undertaken.

\subsection{Pre-planning and project management procedures}

The study also made clear that offshore outsourcing requires considerable preplanning and the identification and formalization of the required processes in 
advance of an approach to potential service providers. Although many of the web-site descriptions suggested that communication and project management procedures and protocols were in-place this was not found to be the case when information transference commenced. This necessitated the implementation of communication and document protocols to manage the process and outsourcing relationship.

\subsection{Upskilling}

The pilot also raised the question of the need for upskilling of service providers in local regulatory conditions. The need for a regulatory and trade referencing library or on-line subscriptions to such services where available is an important aspect that requires consideration in how the process is managed with a service provider. It is difficult to gauge the reality of a firm's capacity in this regard and the progressive costs that may accumulate need to be factored into the project. Should a local practice establish an operating budget to be allocated to upskilling service partners and how is the associated risk managed?

\subsection{Quality assurance and design resolution}

For collaborations to be effective the pilot has indicated that the quality of service provider output is dependant on the quality of the information provided. The use of a highly resolved design development set of drawings in this study has shown the benefits that arise in lessening miscommunication and misunderstanding of design intent, however, these are not obviated in the detailing aspects of the project. It also suggests that significant portions of the design resolution need to be shifted from the construction documentation stage to the design development stage and in the initial relationship with an offshore service provider these need to be controlled in-house. The production of drawings with key review and witness points permits a practice to use the service provider as an important component of their quality assurance system as it forces documentation review, which may not occur in-house due to the convenience of over-the-desk communication. It also highlights the diligence requirements that such arrangements produce, due to the fact that liability cannot be divested to an offshore agency operating with very different legislative controls and the cost associated in pursuing such partners. However, it also indicates that the potential cost differential allows one to become aware of detailed design issues and spend more time developing and resolving the design or pursuing alternate design options.

\subsection{Price as a service indicator}

What has also been alluded to in the study is that cost is not necessarily an indicator of service quality. In this limited study of the two study participants it was the one who was $47 \%$ cheaper that raised more information requests, picked up discrepancies between design development drawings and produced a higher standard of documentation output. 


\section{Conclusion}

The pilot study, though limited in scope still exposed issues that suggest that any decision or evaluation of production outsourcing requires a more holistic approach, linked to determinants other than simply cost. In particular, it has shown that outsourcing is an important exercise for any firm; presenting opportunities but also problems that are not only technical in nature. It forces a rigorous introspective view of one's procedures and methods of work. Each firm will have to organize the work differently, because the elements critical to the practice will change. It is intrinsically linked to the design and production methods of the commissioning practice and the capacity of the service provider to understand and work with these operating methods. Offshore documentation partners cannot be viewed as generic efficient service providers, because the work itself is not generically developed. Distant alliances do represent a different model of project organization. For these alliances to be successful they require the identification of specific collaboration profiles which do not necessarily represent a different model of practice. The next phase of our research hopes to provide some further insights and benchmarks for these collaboration profiles.

\section{References}

[1] PMA., PSMJ A/E Automation and Information Technology Survey, Practice Management Associates, Dedham, Massachusetts, 2001.

[2] ZweigWhite., ZweigWhite Successful Firms Survey of $A / E / P$ and Environmental Consulting Firms, Natick, Massachusetts, 2001.

[3] Carmel, E. and Agarwal, R. The Maturation of Offshore Sourcing of Information Technology Work, MIS Quarterly Executive, Vol. 1, No. 2, pp. 65-76, June 2002.

[4] Christopherson, S., Behind the scenes: How transnational firms are constructing a new international division of labor in media work, Geoforum, (In press), www.sciencedirect.com, June 2006.

[5] Tombesi, P., Dave, B., Gardiner, B., Scriver, P., Evaluating the industrial potential of digital outsourcing in architecture; Methodological challenges and choices, CIB W096 Architectural Management Meeting, Designing Value: New Directions in Architectural Management, Danish Technical University, Lyngby, pp. 441-450, 2005.

[6] Tombesi, P., Dave, B., Gardiner, B., Scriver, P., Rules of engagement: Testing the attributes of distant outsourcing marriages, Journal of Architectural Engineering and Design Management, (accepted for publication 2006). 